\title{
Noninvasive study of structural and functional features of neonatal skin
}

\author{
I. L. Shlivko*, , V. A. Kamensky ${ }^{\dagger}$, M. Y. Kirillin ${ }^{\dagger}$, P. D. Agrba ${ }^{\dagger}$, \\ O. E. Garanina*, M. S. Neznakhina*, D. O. Ellinsky*, \\ A. S. Maksimova* and E. V. Donchenko* \\ *Nizhny Novgorod State Medical Academy \\ 603005 Minin and Pozharsky Square \\ 10/1, Nizhny Novgorod, Russia \\ ${ }^{\dagger}$ Institute of Applied Physics RAS \\ 603950 Ulyanov str. 46, Nizhny Novgorod, Russia \\ $\$$ Nizhny Novgorod Research Institute of \\ Traumatology and Orthopedics \\ 603155 Verkhnevolzhskaya Embankment \\ 18, Nizhny Novgorod, Russia \\ \$irshlivko@gmail.com
}

Received 9 August 2013

Accepted 29 October 2013

Published 12 December 2013

\begin{abstract}
In the present paper, we describe the first complex multifocal noninvasive morphological and functional study that enabled us to define specific qualitative and quantitative features of neonatal skin. A complex morphofunctional examination of 10 infants aging from 1 to 28 days was performed by optical coherence tomography (OCT) device with a flexible probe at the wavelength of $920 \mathrm{~nm}$ with longitudinal resolution of $20 \mu \mathrm{m}$ and transverse resolution of $25 \mu \mathrm{m}$ with simultaneous measurement of skin functional parameters. The OCT images of neonatal thin skin have organized layered structure with four horizontally oriented layers. Thick skin of newborns has no structure typical for adult skin and no clear transition from the papillary to the cellular dermis. Thus, we show for the first time to our knowledge that neonatal thick skin differs structurally and functionally from adult skin. Structurally, it differs by a loose arrangement of stratum corneum squamae and thinner epidermis and papillary layer of dermis. The functional differences are lower transepidermal water loss, localization-dependent humidity, higher erythema level, and lower pigmentation. The principal structural differences between neonatal and adult skin show that skin structure formation is not completed by the moment of birth.
\end{abstract}

Keywords: Optical coherence tomography; skin; skin barrier; neonate.

This is an Open Access article published by World Scientific Publishing Company. It is distributed under the terms of the Creative Commons Attribution 3.0 (CC-BY) License. Further distribution of this work is permitted, provided the original work is properly cited. 


\section{L. Shlivko et al.}

\section{Introduction}

Clinically, infant skin is frequently described as soft and tender, at the same time it is considered to be delicate, thin and susceptible to dermatitises and infections. The protective skin functions are formed from the early stages of human life. Infancy, neonatal period in particular, is the time when skin is adapting from a humid and warm environment with constant temperature to a dry gaseous environment with temperature variations. Study of the functional skin parameters, such as transepidermal water loss (TEWL), stratum corneum humidity, erythema and pigmentation gives an insight into the mechanisms responsible for the formation of a mature cutaneous covering. The barrier function and transport characteristics of the neonatal stratum corneum differ from those in adults and change substantially during the first year of life. It was found ${ }^{1}$ that the infant stratum corneum has a higher water content, loses and accumulates water faster in normal state and at rest. The known features of the functional structure are evidently attributed to the features of neonatal skin microstructure. Until recently, the investigation of the morphological features of neonatal skin was restricted for ethical reasons due to invasiveness of the analysis.

The light-tissue interaction in the visible wavelength range is broadly used in infant intensive care, for instance, for transcutaneous bilirubinometry or oximetry. Information about optical properties of neonatal skin is very limited, despite the fact that the majority of optical diagnostic methods in neonatology are immediately connected with the lightskin interaction. The optical properties of neonatal skin were studied by Saidi et al. ${ }^{2}$ who measured the absorption and scattering coefficients as a function of age and found that the scattering coefficient increases with age as a result of skin maturation. The measurements were performed in a test tube, hence, the optical properties could differ from those measured in vivo owing to inevitable typical procedures of tissue sampling preparation.

The optical properties of neonatal skin as a function of age and phototype were investigated by a group of scientists from the Netherland Center for Biomedical Engineering and Physics. ${ }^{3}$ The absorption $\left(\mu_{\mathrm{a}}\right)$ and scattering $\left(\mu_{\mathrm{s}}\right)$ coefficients in neonatal skin were measured in natural conditions at the wavelength between 450 and $600 \mathrm{~nm}$. The values of $\mu_{\mathrm{a}}$ and $\mu_{\mathrm{s}}$ were registered at four localizations: forehead, breast bone, arm and leg in 60 newborns of different gestational age, postnatal age and skin pigmentation. It was found that the absorption and scattering coefficients are almost independent of localization of skin section or gestational age, but strongly depend on neonatal skin pigmentation.

Active development of novel noninvasive technologies enables up-to-date studies of functional and structural features of neonatal skin in vivo.

The first work on noninvasive investigation of infant skin at the age of 3 months and older using the method of confocal microscopy was done in $2010 .{ }^{4}$ It was found that dermal papillae are distributed quite uniformly in infant skin, in contrast to adult skin where papillae vary both in size and shape. The neonatal stratum corneum and suprapapillary epidermis are $30 \%$ thinner than the adult ones. The stripped stratum corneum permitted assessing the size of corneal scales that also proved to be smaller in newborn infants.

In the present paper, we describe the first complex multifocal noninvasive morphological and functional study that enabled us to define specific qualitative and quantitative features of newborn infants. The structure of neonatal skin in real conditions was studied by optical coherence tomography (OCT) which allows noninvasive imaging of studied skin sections at a depth up to $1 \mathrm{~mm}$.

The goal of the study is investigation of the structural and functional features of neonatal skin.

\section{Materials and Methods}

A total of 10 newborn term babies ( 2 females and 8 males) aging 1 to 28 days underwent complex multifunctional examination. A total of 10 teenagers (4 females and 6 males) at the age from 12 to 15 years old were examined for comparison. All subjects were healthy. The functional and structural skin parameters were investigated at 14 points in different anatomic areas: hairline, center of the forehead, cheekbone, angle of eye, angle of mouth, shoulder, extensor and flexor surface of forearm, palm, umbilicus, jugular fossa, shin, foot rear and heel.

The teenagers and newborn infants were examined with approbation from the parents who signed written informed consent. The study was approved by the local ethic committee of the Nizhny Novgorod Research Institute of Dermatovenerology (Protocol No. 12 from 01.10.04). 
Morphological skin state was studied by an OCT device with a removable flexible probe with microscanner produced at the Institute of Applied Physics (IAP RAS, Nizhny Novgorod, Russia), with the following characteristics: central probing wavelength is $920 \mathrm{~nm}$, radiation source power at the probe output is $1.5 \mathrm{~mW}$, axial and transversal resolutions are is 20 and $25 \mu \mathrm{m}$, respectively, maximal scanning depth is $1.5 \mathrm{~mm}$, imaging rate is $1 \mathrm{fps}^{5}$

The study was carried out without immersion to exclude its influence on optical and functional parameters of skin. The force and time of OCT probe pressing to skin surface were controlled during the study to exclude the impact of pressure on the characteristics of the obtained OCT images. ${ }^{6}$

In order to avoid the effect of OCT-image noise on the measured parameters of the skin we averaged the OCT A-scans in transversal directions over the areas without manifested inhomogeneities. The following parameters were extracted from the averaged Ascans for skin characterization: contrast between the layers in the OCT image, average optical thickness of each distinguished layer and OCT imaging depth (OID) which was defined as the maximal depth at which the averaged OCT signal exceeds the average noise level for $5 \mathrm{~dB}$. The two former parameters were calculated only for those OCT images where layered structure was manifested: thick and thin skin of teenagers and thin skin of neonates. In order to convert optical distances into physical ones the measured values were normalized by an average refractive index value taken as 1.42. More detailed description of OCT-images processing algorithm can be found Ref. 5. Due to axial resolution of the OCT system equal to $20 \mu \mathrm{m}$ (full width at half maximum (FWHM) of source coherence function), the accuracy of thickness measurement can be evaluated as $7 \mu \mathrm{m}$ (half width at half maximum (HWHM) of source coherence function normalized for 1.42).

A total of 280 OCT images of neonatal and teenage skin were obtained and processed in the study. Functional skin characteristics, such as stratum corneum humidity, TEWL, pigmentation and erythema were measured by means of a multifunctional Multi SkinTest Center MC 750 (Courage + Khazaka Electronic GmbH, Germany).

Statistical analysis of the obtained data was performed in order to understand the significance of difference between groups of neonates and teenagers and significance of parameters variation within each group. The Shapiro-Wilk test was used as a normality test. The Mann-Whitney test and Friedman rank test (Friedman ANOVA) were used to check the significance of parameters variation within a group and between groups, respectively. The statistical significance was taken to be 0.05 .

\section{Results and Discussion}

Typical OCT image of neonatal thin skin is presented in Fig. 1. Analysis of this image shows organized layered structure of thin neonatal skin featuring the several layers. The top layer [see 1 in Fig. 1(a)] is a bright, strongly scattering surface layer of variable thickness corresponding to superficial part of stratum corneum represented by loose dry cells. Variation in thickness associates with random localization of strongly scattering single dry cells contributing to the OCT signal. The 2nd layer [see 2 in Fig. 1(a)] is a darker, homogeneous, weakly scattering layer of approximately constant thickness corresponding to cellular layers of epidermis and to the zone of epidermal-dermal junction. The 3rd layer [see 3 in Fig. 1(a)] is manifested by a bright, strongly scattering layer of variable thickness corresponding to reticular derma smoothly passing to the 4th layer [see 4 in Fig. 1(a)], a weakly scattering, inhomogeneous layer; the OCT signal level is gradually decreasing with depth that corresponds to the lower level of reticular derma with a large number of inclusions.

We could not identify other layers in the lower areas as the signal level dropped. Vessels of different diameter, glands or hair bulbs localized at different levels were visualized as round, oval or striped patterns with distinct boundaries.

Comparative analysis of stratum corneum thickness between neonates and teenagers groups was performed qualitatively due to limited axial resolution of the employed OCT system which does not allow to accurately measure this value. Qualitative analysis allow concluding that in neonate skin the thickness of superficial stratum corneum layer and corresponding OCT signal level are higher compared to those for teenagers (see Fig. 1).

Taking into consideration that the stratum corneum is an integral part of the epidermis and the total thickness of the infant epidermis is smaller than of the adult epidermis, we describe rather qualitative than quantitative changes of the stratum corneum. In this case, the observable increase in the cellular layer fraction is the result of loose 


\section{L. Shlivko et al.}
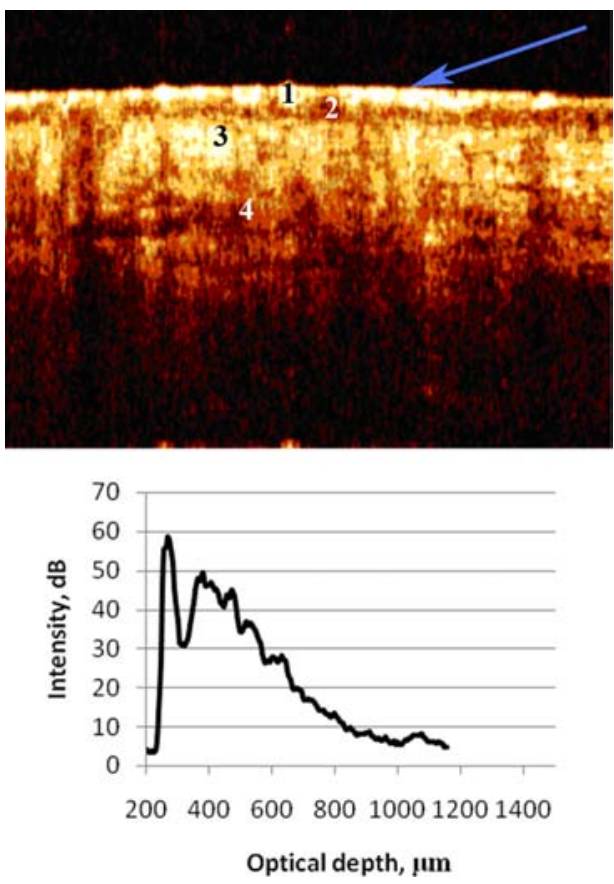

(a)
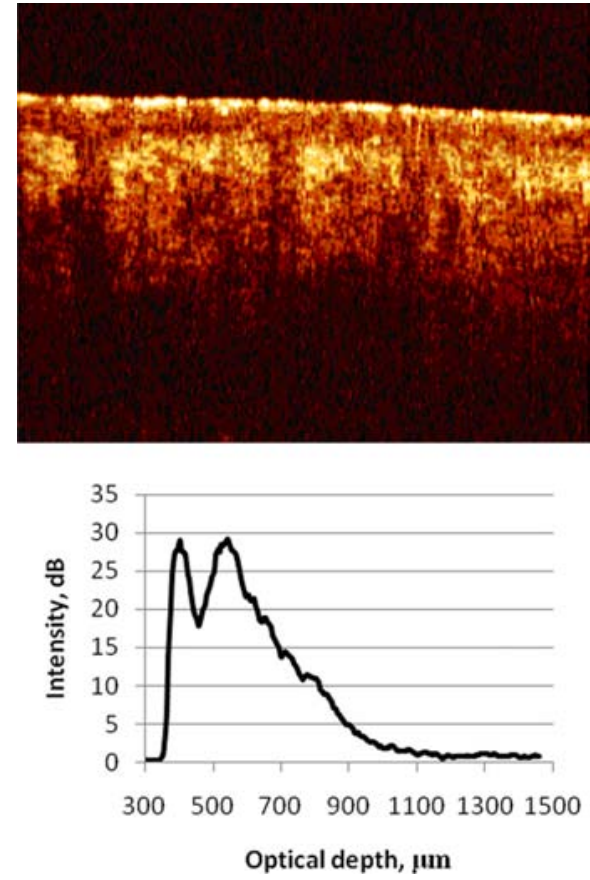

(b)

Fig. 1. OCT images and averaged A-scans of skin localized at the face (cheek bone) of a 13-days old newborn (a) and a teenager 14 years old (b).

arrangement of the scales, their thickness and the air filling in the space between them.

The OCT images of palms and soles are also qualitatively different. For example, neonates have no typical pattern of thick skin with a pronounced stratum corneum and papillae. The OCT image of thick skin of newborn infants has no typical layered structure, contrast and distinguished boundaries between the layers, which makes numerical processing of these images impossible (see Fig. 2).

Investigation of structural skin parameters revealed that neonatal epidermis is thinner than teenage epidermis, with the value of parameters statistically less significant at the points of hairline, angle of mouth, umbilicus and foot rear. The minimal size of epidermis in both groups was recorded in the angle of eye (see Table 1).

Analysis of the results of functional examination demonstrated that humidity in the test and reference groups greatly varies depending on localization. It was found that stratum corneum hydration in the face skin of newborns is lower, except for the angle of mouth. The palms of newborns have maximum humidity among all considered localization with the median value of 61 corneometric units (c.u.) with 1st quartile-3rd quartile interval of
[44;74], which is statistically significantly higher compared to that in teenagers $(p=0.0025)$. In the area of shoulder, forearm, umbilicus, heel and foot rear the humidity for newborns is also statistically significantly higher than that for teenagers (see Table 2). Here in after when comparing two values we mean statistically significant difference.

TEWL in newborns is less than in teenagers in the majority of cases. In the area of leg, palm and heel, TEWL has close values $(p=0.9)$, and TEWL of foot rear skin of infants is higher than in teenagers.

Infant erythema was found to be higher at all considered localizations and the difference in the erythema level is statistically significant at all points, except the angle of mouth. The highest absolute values of erythema were revealed in the palms of newborns with median value of $46[43 ; 57]$ a.u. and soles with median value of $47[41 ; 56]$ a.u., whereas almost lowest erythema levels were registered in same localizations in teenagers.

Pigmentation is lower in newborns compared to teenagers at the most of considered localizations, except center of forehead, angle of eye, palm, hairline and cheek bone (see Table 2).

The Friedman rank test showed that all the parameters studied in our research have pronounced 

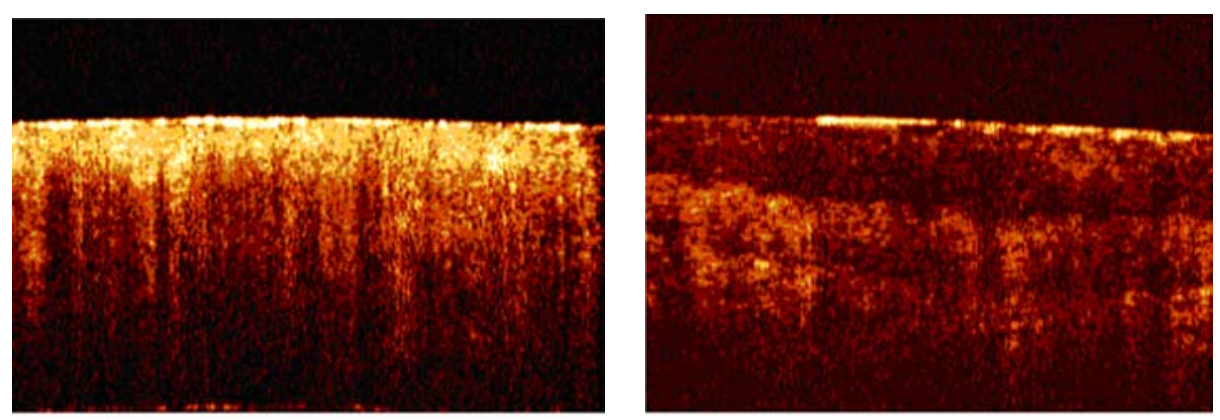

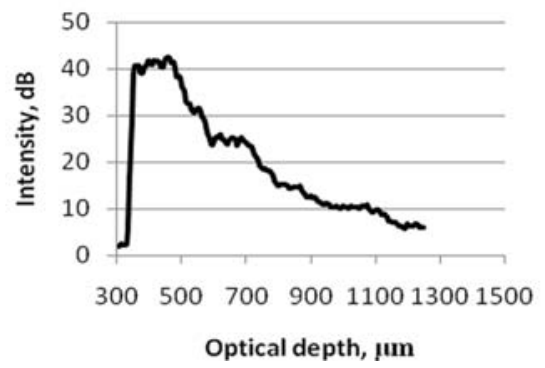

(a)

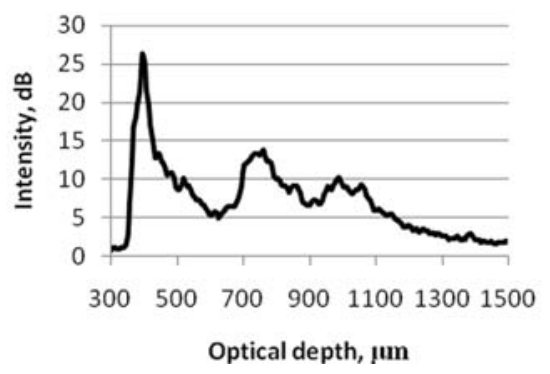

(b)

Fig. 2. OCT images and A-scans of thick palm skin of a newborn (a) and a teenager (b).

individual distinctions $(p<0.000001)$. An exception is the value of OID in neonatal skin $(p<0.1)$.

During the first year of life, infant skin experiences manifested changes in the course of maturation. ${ }^{7}$ The most pronounced skin alterations are associated with the processes of water holding and loss. ${ }^{1,8}$ The revealed features of the functional structure are evidently explained by the peculiarities of neonatal skin microstructure.

Our study showed that a strongly scattering surface layer appears in OCT images of neonatal skin. The strongly scattering top layer is presumably attributed to the specific features of the infant stratum corneum structure, in particular, to loosely arranged squamae. In addition to the loose organization of squamae, their thickness may also play a definite role as it is higher than in adults, despite its smaller size. ${ }^{9}$

However, in general, the organized layered structure in the OCT image of neonatal thin skin fully repeats that of teenager skin. At the same time, study of palm and sole skin revealed dramatic differences between thick skin of newborns and adults. Neonatal thick skin has no layered structure, which is indicative of an incomplete process of skin formation.

The OID strongly depends on the surface layer of the studied tissue. Due to strongly scattering stratum corneum and in the absence of immersion that could match refractive indices, OID in newborns is expected to demonstrate lower values compared to teenagers, whereas our study demonstrates the opposite results. The volume, density and diameter of collagen fibers are known to be minimal up to the age of one year. ${ }^{10}$ This observation was confirmed ${ }^{4}$ by confocal laser scanning microscopy study which demonstrated that, in contrast to adults, infant skin has no obvious transition from the papillary to the cellular dermis. The specific features of the structure of dermal fibers evidently play the decisive role in light penetration deep into the tissue. The revealed differences in the anatomic structure are traced in the functional state of neonatal skin. For example, TEWL correlates with the thickness of stratum corneum and epidermis. Loosely arranged and thick squamae in case of thin epidermis allow waterholding and prevent its evaporation.

Taking into consideration the results of humidity measurements we can assume that in the first days after birth, open skin sections of newborns dry out under the action of external factors, unlike the sections protected by clothing. The exception is the angle of mouth, where high humidity is evidently connected with the functional load of the skin in this area. More pronounced erythema is explained by thinner epidermis, hence, by a smaller distance between vessels and skin surface. This hypothesis was confirmed by the calculations which showed an 


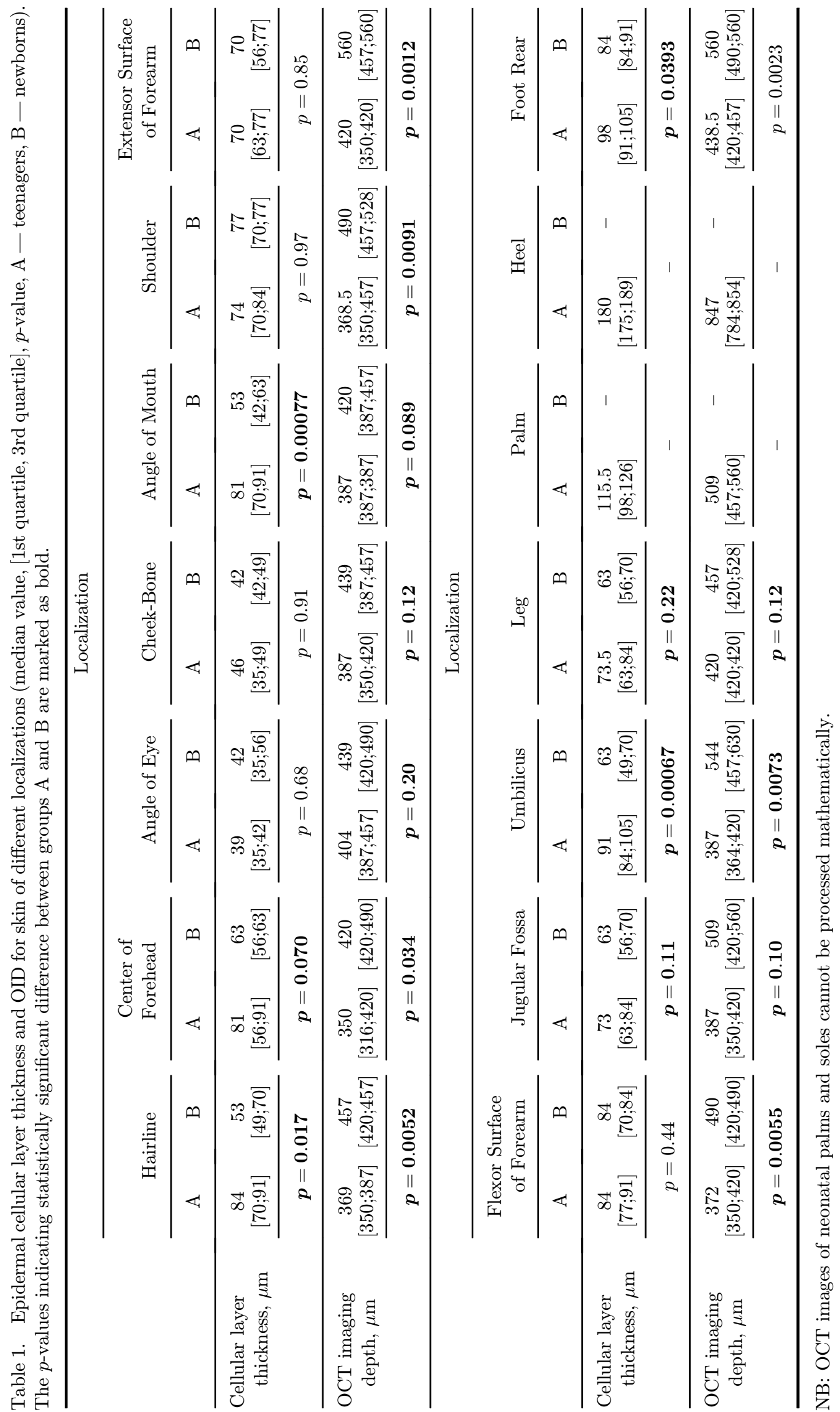


Noninvasive study of structural and functional features of neonatal skin

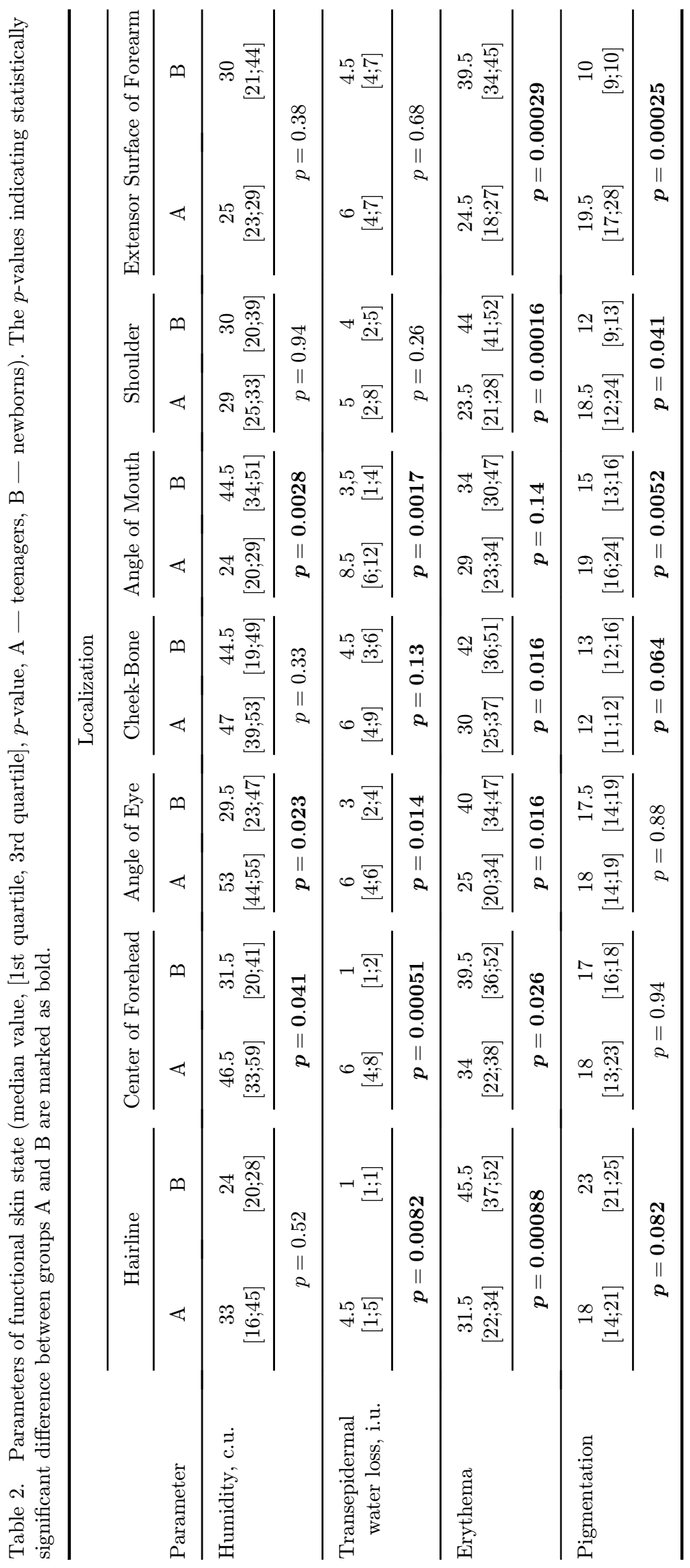




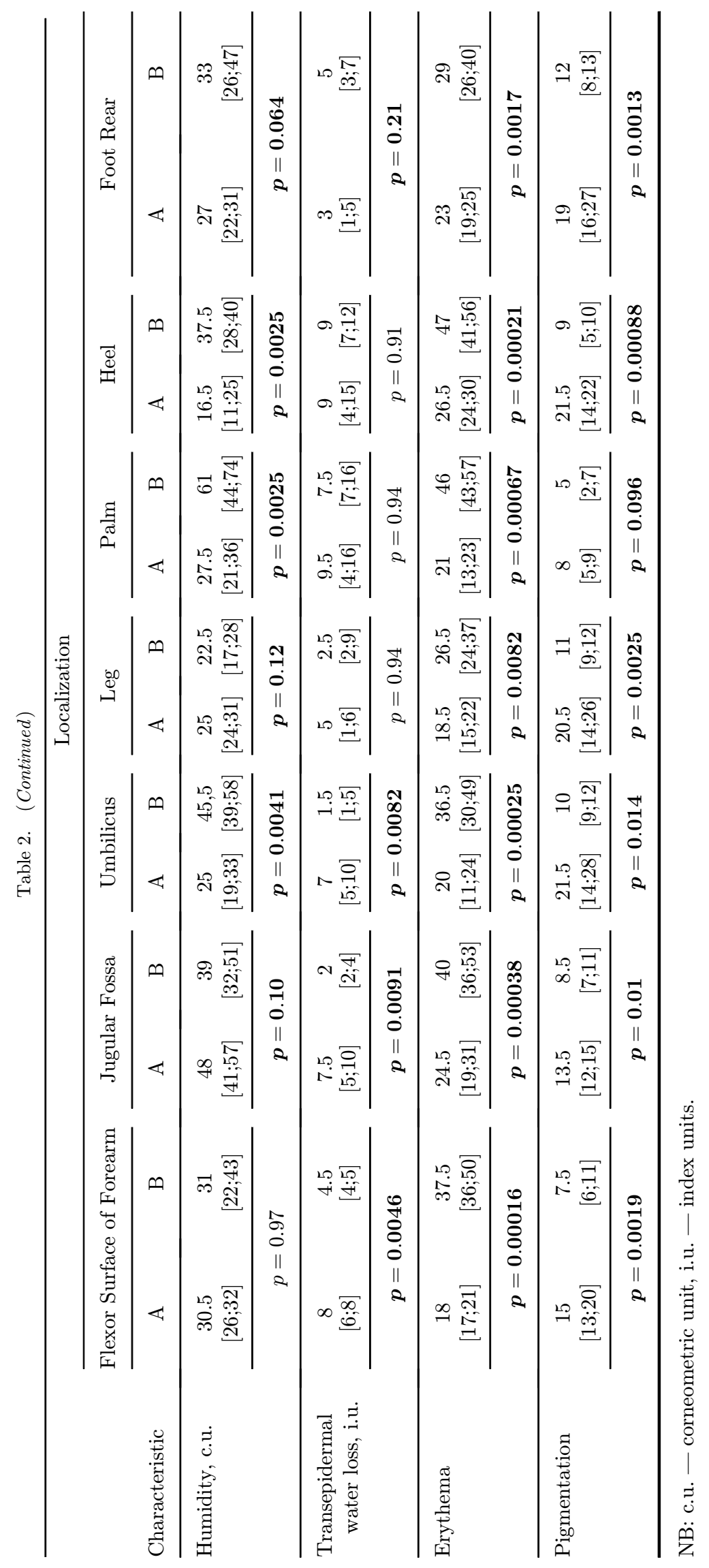




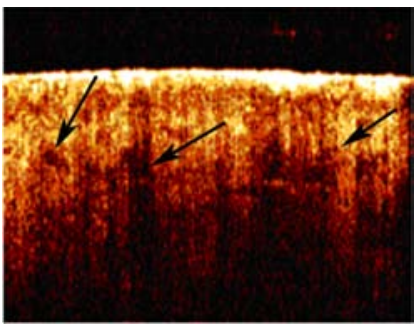

(a)

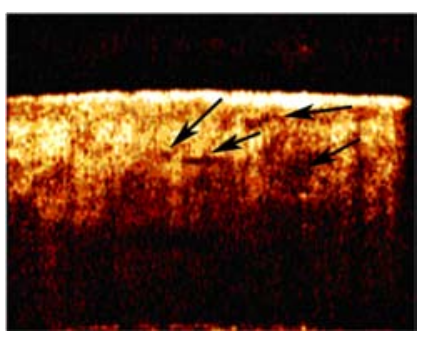

(d)

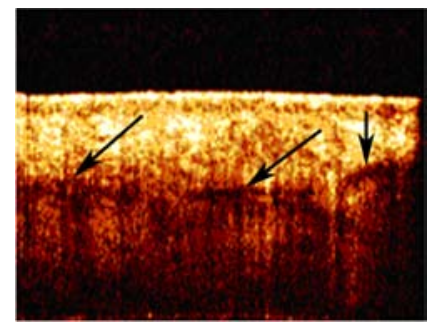

(b)

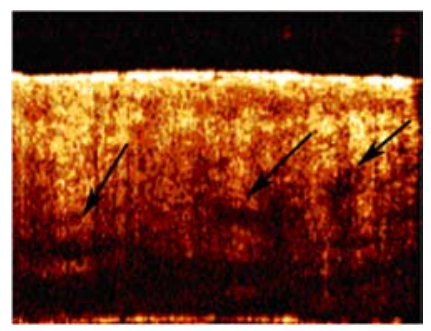

(e)

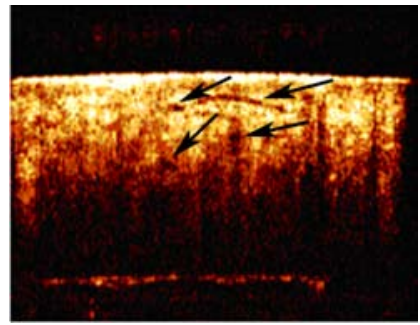

(c)

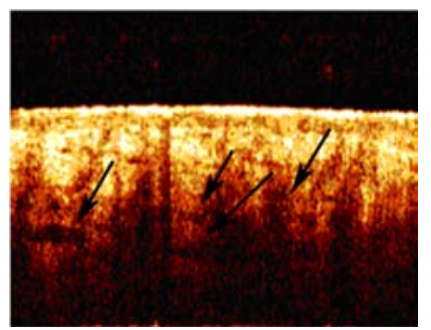

(f)

Fig. 3. OCT image of neonatal skin of different localizations, the arrows show vessels in the 3rd and 4th layers (a-f).

inverse mean highly significant correlation between the thickness of epidermis and the level of erythema. At the same time, specific features of vasomotor control and precapillary sphincter paresis may also be responsible for a high level of erythema in newborns. On the other hand, we recorded multiple rounded patterns of different diameter, probably corresponding to dermal vascular plexuses (see Fig. 3), in all tomograms of neonatal skin with high OID.

\section{Conclusion}

To conclude, we show that the OCT images of neonatal thin skin have organized layered structure with four horizontally oriented layers. For the first time to our knowledge we demonstrated that thick skin of newborns has no structure typical for adult skin and no clear transition from the papillary to the cellular dermis, which proves that formation of thick skin structure continues in newborns after birth. Neonatal skin differs structurally and functionally from teenager skin. Structurally, it differs by a loose arrangement of stratum corneum squamae and thinner epidermis and papillary layer of dermis. The functional differences are lower TEWL, localization-dependent humidity, higher erythema level and lower pigmentation. The basic structural differences revealed in thick skin demonstrate an incomplete process of skin structure formation by the moment of birth.

\section{Acknowledgments}

The work is financially supported by the Ministry of Education and Science of Russian Federation (project 8741) and RFBR (project 13-02-97092).

\section{References}

1. J. Nikolovski, G. N. Stamatas, N. Kollias, B. C. Wiegand, "Barrier function and water-holding and transport properties of infant stratum corneum are different from adult and continue to develop through the first year of life," J. Invest. Dermatol. 128(2), 1728-1736 (2008).

2. I. S. Saidi, S. L. Jacques, F. K. Tittel, "Mie and Rayleigh modeling of visible-light scattering in neonatal skin," Appl. Opt. 34(31), 7410-7418 (1995).

3. N. Bosschaart, R. Mentink, J. H. Kok, T. G. van Leeuwen, M. C. Aalders, "Optical properties of neonatal skin measured in vivo as a function of age and skin pigmentation," J. Biomed. Opt. 16(9), 097003 (2011).

4. G. N. Stamatas, J. Nikolovski, M. A. Luedtke, N. Kollias, B. C. Wiegand, "Infant skin microstructure assessed in vivo differs from adult skin in organization and at the cellular level," Pediatr. Dermatol. 27(2), 125-131 (2010).

5. I. L. Shlivko, G. A. Petrova, M. V. Zor'kina, O. E. Tchekalkina, M. S. Firsova, D. O. Ellinsky, P.D. Agrba, V.A. Kamensky, E.V. Donchenko, "Complex assessment of age-specific morphofunctional features 


\section{L. Shlivko et al.}

of skin of different anatomic localizations," Skin Res. Technol. 19(1), 85-92 (2013).

6. M. Y. Kirillin, P. D. Agrba, V. A. Kamensky, "In vivo study of the effect of mechanical compression on formation of OCT images of human skin," $J$. Biophotonics. 3(12), 752-758 (2010).

7. N. J. Evans, N. Rutter, "Development of the epidermis in the newborn," Biol. Neonate. 49(2), 74-80 (1986).

8. M. O. Visscher, R. Chatterjee, J. P. Ebel, A. A. LaRuffa, S. B. Hoath, "Biomedical assessment and instrumental evaluation of healthy infant skin," Pediatr. Dermatol 19(6), 473-481 (2002).
9. N. Kashibuchi, Y. Hirai, K. O’Goshi, H. Tagami, "Three-dimensional analyses of individual corneocytes with atomic force microscope: Morphological changes related to age, location and to the pathologic skin conditions," Skin Res. Technol. 8 (4), 203-211 (2002).

10. L. Vitellaro-Zuccarello, S. Cappelletti, V. D. P. Rossi, M. Sari-Gorla, "Stereological analysis of collagen and elastic fibers in the normal human dermis: Variability with age, sex, and body region," Anat. Rec. 238(2), 153-162 (1994). 